\title{
High Impedance Droplet-Solid Interface Lipid Bilayer Membranes
}

\author{
Xuejing Wang, ${ }^{\dagger}$ Shenghua $\mathrm{Ma},{ }^{\dagger}$ Yingchun Su, ${ }^{\dagger}$ Ying Zhang, ${ }^{\dagger}$ Hongmei Bi, ${ }^{\dagger}$ Lixue Zhang, \\ and Xiaojun $\mathrm{Han}^{*}{ }^{\dagger}$ \\ ${ }^{\dagger}$ State Key Laboratory of Urban Water Resource and Environment, School of Chemical Engineering and Technology, Harbin \\ Institute of Technology, No. 92 West Da-Zhi Street, Harbin 150001, China \\ ‡Qingdao Key Lab of Solar Energy Utilization and Energy Storage Technology, Qingdao Institute of Bioenergy and Bioprocess \\ Technology, Chinese Academy of Sciences, No. 189 Songling Road, Qingdao 266101, China
}

Supporting Information

ABSTRACT: A droplet-solid interface lipid bilayer membrane (DSLM) with high impedance was developed through controlling the contact area between an aqueous droplet and electrode. The electrode size can be easily controlled from millimeter to micrometer level. The droplet-solid interface lipid bilayer membranes were characterized by cyclic voltammetry (CV), electrochemical impedance spectroscopy (EIS), and fluorescence microscopy. The fluorescence recovery after photobleaching (FRAP) was applied to determine the diffusion coefficient of egg PC DSLM to be $2.58 \mu \mathrm{m}^{2}$ $\mathrm{s}^{-1}$. The DSLM resistance can reach up to $26.3 \mathrm{G} \Omega$, which was then used to study the ion channel behavior of melittin. The resistivity of

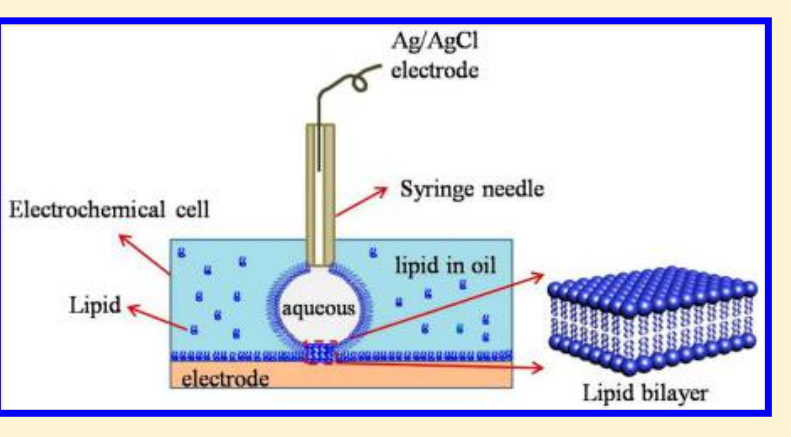
the bilayer membrane decreased linearly with the increase of melittin concentration in the membrane. The high impedance and fluidity of DSLM makes it an ideal model cell membrane system for ion channel study and high-throughput drug screening.

$\mathrm{M}$ embrane proteins, especially ion channels and receptor proteins, are vital components of biomembranes for signaling, mass transport, and immune recognition. It is estimated that about $50 \%$ of pharmaceutical drugs in the market target membrane proteins. ${ }^{1}$ Black lipid membranes (BLMs) have been widely used for detecting single channel activities of pore-forming peptides, ion channels, ion pumps, and receptors. $^{2-8}$ However, the drawback of this artificial biomembrane system is low throughput caused by its delicate preparation procedure and short lifetime. To improve the stability of the artificial biomembranes, several novel mimic biomembrane models have been invented. Since Hennesthal et al. ${ }^{9}$ pioneered in preparing lipid bilayer on nanopores, many research groups have focused on lipid bilayer formation on porous materials such as porous alumina, ${ }^{10-14}$ porous silicon nitride, ${ }^{15,16}$ porous silicon, ${ }^{17,18}$ etc., to enhance the stability of biomembranes. Generally, the resistance of the bilayer prepared on nanopores can be $G \Omega$ magnitude, which is 3-6 orders higher than that in black lipid membranes (BLMs). Thus, nanopore lipid bilayers were applied to study membrane proteins or peptides such as gramicidin, ${ }^{13,16} \alpha$-hemolysin, ${ }^{12}$ and melittin. ${ }^{19}$ Recently, a gigaseal self-assembled long-lifetime lipid bilayer was prepared on polyelectrolyte multilayer-filled nanopores ${ }^{19}$ by Sugihara et al. The lifetime of this high impedance ( $1 \mathrm{G} \Omega$ ) lipid bilayer can be as stable as 2.5 weeks to monitor the activity of a single peptide channel. However, the success rate of the gigaseal was only $30 \%$, and the normalized resistivity calculated from the pore area was just $5 \Omega \mathrm{cm}^{2}$. In summary, the nanopore-supported lipid bilayers own good stability and fluidity which are beneficial for the investigation of ion channels. However, nanopore arrays usually need a complicated fabrication process and are generally expensive. As an alternative, a supported lipid bilayer (SLB), ${ }^{20-25}$ a planar structure sitting on a solid support, prolongs membrane lifetime. However, the electrode area is normally so large that it makes the roughness problem prominent, which leads to large current leakage. Droplet interface bilayers (DIBs) ${ }^{26,27}$ are another type of novel artificial lipid bilayer made by contacting aqueous droplets in an oil solution in the presence of phospholipids. Compared with the planar bilayers created with the Montal-Mueller method or the black lipid membranes, DIBs are a simple way to generate stable bilayers suitable for the detection of membrane proteins. In addition to the formation of a lipid bilayer between two monolayer-coated aqueous droplets, a bilayer between an aqueous droplet and an agarose hydrogel-coated glass coverslip has been prepared and used to detect membrane channels by Wallace's group. ${ }^{28}$ So far, there has been no report on the formation of a lipid bilayer between the aqueous droplet and the solid support.

Herein we took the advantages of SLBs and DIBs and proposed a novel droplet-solid interface lipid bilayer membrane (DSLM) with high impedance by controlling the contact area between the aqueous droplet and the conductive solid substrate in an oil solution containing phospholipids (Figure 1). An Ag/AgCl wire reference electrode was inserted

Received: August 7, 2014

Accepted: January 20, 2015

Published: January 20, 2015 


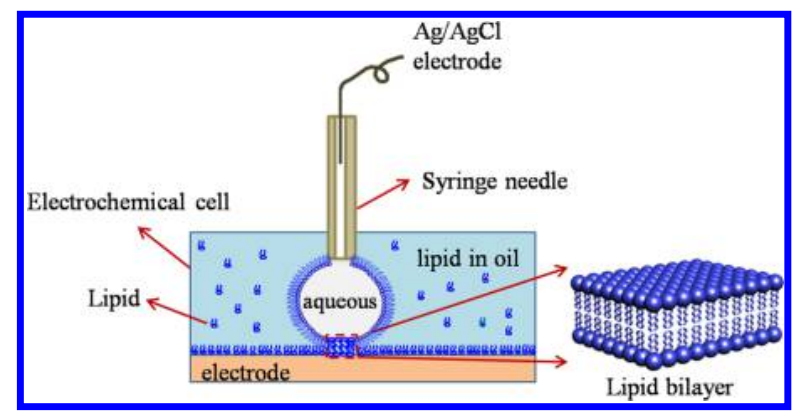

Figure 1. Schematic representation of the droplet-solid interface lipid bilayer membranes.

into the syringe for the electric property measurements of the lipid membranes. In addition, the ion channel property of melittin was measured using this artificial membrane system by electrochemical impedance spectroscopy (EIS). This high impedance lipid bilayer membrane system is easy to prepare, low cost, fluid, and highly stable, which gives it great potential for drug screening.

\section{EXPERIMENTAL SECTION}

Materials. Egg yolk L- $\alpha$-phosphatidylcholine (egg PC) and 1,2-diphytanoyl-sn-glycero-3-phosphocholine (DPhyPC) were obtained from Avanti Polar Lipids. Trimethoxy(octadecyl)silane (TODS), chloroform, potassium ferricyanide $\left(\mathrm{K}_{3} \mathrm{Fe}\right.$ $\left.(\mathrm{CN})_{6}\right)$, and melittin $(\geqslant 97 \%)$ were purchased from SigmaAldrich (China). Glass slides coated with indium tin oxide (ITO, sheet resistance $\approx 8$ to $12 \Omega /$ square, thickness $\approx 160 \mathrm{~nm}$ ) were produced by Hangzhou Yuhong Technology (China). Texas red-labeled 1,2-dihexadecanoyl-sn-glycero-3-phosphoethanolamine triethylammonium salt (TR-DHPE) was obtained from Invitrogen (China). Potassium chloride, potassium dihydrogen phosphate, dipotassium hydrogen phosphate trihydrate, toluene, and $n$-hexane were supplied by Xilong Chemicals (China). The PBS $(\mathrm{pH}=7.4)$ buffer was a mixture of $\mathrm{KH}_{2} \mathrm{PO}_{4}$ and $\mathrm{K}_{2} \mathrm{HPO}_{4} \cdot 3 \mathrm{H}_{2} \mathrm{O}$ (mass ratio = 19:81). Ethanol was purchased from FuYu Chemicals (China). Millipore MilliQ water with a resistivity of $18.0 \mathrm{M} \Omega \cdot \mathrm{cm}$ was used for solution preparation in the experiments.

Experimental Setup. The experimental setup is illustrated in Figure 1. A modified syringe with a silicon blunt needle coated with polyimide (inner diameter $=50 \mu \mathrm{m}$ ) was used to control the size of the aqueous droplet in the oil solution on the surface of ITO electrodes. Considering the small current during the measurement, a two-electrode system was applied to characterize the lipid membrane system. In other words, the ITO electrodes are working electrodes, while an $\mathrm{Ag} / \mathrm{AgCl}$ wire acts as both reference and counter electrode. The fabrication of the $\mathrm{Ag} / \mathrm{AgCl}$ wire reference electrode as well as its calibration (Figure S1) is described in Supporting Information.

All electrochemical experiments were performed using an Autolab electrochemical workstation (PGATAT320N, Switzerland). Cyclic voltammetry (CV) and electrochemical impedance spectroscopy (EIS) were used to characterize the formation of DSLM. EIS measurements were recorded within a frequency range of $10^{-2}$ to $10^{5} \mathrm{~Hz}$ with a signal amplitude of $100 \mathrm{mV}$. The electrolyte was $0.2 \mathrm{M}$ PBS buffer $(\mathrm{pH}=7.4$ ) containing $0.5 \mathrm{mM} \mathrm{K}_{3} \mathrm{Fe}(\mathrm{CN})_{6}$ and $2 \mathrm{M} \mathrm{KCl}$.

Preparation of TODS-Modified ITO Substrate. The $1 \times$ $1 \mathrm{~cm}^{2}$ ITO pieces were sonicated in detergent, distilled water, and ethanol each for $15 \mathrm{~min}$, successively. After drying under a stream of nitrogen, the ITO slides were put into plasma cleaner (Diener electronic, Zepto) for $30 \mathrm{~s}$ to remove trace organic compounds. Then the ITO electrodes were silanized by incubation in $1 \mathrm{vol} \%$ TODS toluene solution for $4 \mathrm{~h}$ followed by rinsing with toluene and ethanol and finally nitrogen-drying.

Formation of Droplet-Solid Interface Lipid Bilayer Membranes. The plasma-treated bare ITO electrodes were covered with $0.25 \mathrm{mg} / \mathrm{mL}$ lipid hexane solution for $1 \mathrm{~h}$ to form a lipid monolayer on its surface, as shown in Figure 1. The aqueous droplets were extruded from the syringe and left hanging for 10-15 min. The lipid monolayer formed spontaneously at the surface of the droplet with the polar head of the lipid molecule toward the inner aqueous solution and the hydrophobic tail toward the oil phase. Afterward the droplet was moved carefully down to the surface of bare ITO electrode to allow the bilayer membrane formation. The in situ observation of the droplet on the ITO surfaces was performed using a high resolution camera.

Fluorescence Recovery after Photobleaching (FRAP). A Nikon 80i fluorescence microscope equipped with a Nikon DS-Fil digital camera was used to image the lipid bilayer membranes. The FRAP data were analyzed by evaluating intensity with ImageJ. The lateral diffusion coefficient was calculated from $D=0.224 \omega^{2} / t_{1 / 2}$, where $\omega$ is the radius of the bleached spot and $t_{1 / 2}$ is the half-life of fluorescence recovery. ${ }^{29,30}$

\section{RESULTS AND DISCUSSION}

Contact Area Control between Droplets and Solid Substrates. Conventionally, the electrode is immersed in an aqueous solution for electrochemical measurements. Here we controlled the electrode area by manipulating the contact area between an aqueous droplet and electrode. Using this method, the electrode size can be easily controlled from millimeter to micrometer range. The contact area control was conducted between droplets and TODS (trimethoxy(octadecyl)silane)modified ITO slides. The electrode size can be adjusted down to $\sim 30 \mu \mathrm{m}$ in radius. Figure $2 \mathrm{~A}$ shows the images of droplets on TODS-modified ITO slides with different effective electrode sizes, which can be easily controlled by manipulating the droplets. Due to the mirror effect of ITO electrodes, the silhouettes of droplets can also be seen in Figure 2A. The cyclic voltammetry $(\mathrm{CV})$ curves $\mathrm{a}, \mathrm{b}$, and $\mathrm{c}$ in Figure $2 \mathrm{~B}$ correspond to the droplet situations $\mathrm{a}, \mathrm{b}$, and $\mathrm{c}$ in Figure 2A, respectively. The CV measurements were performed in aqueous solution containing $1 \mathrm{mM} \mathrm{K} \mathrm{K}_{3} \mathrm{Fe}(\mathrm{CN})_{6}$ and $2.5 \mathrm{M} \mathrm{KCl}$ with a scanning rate of $20 \mathrm{mV} / \mathrm{s}$. The shapes of the $\mathrm{CV}$ curves change from peak shape to " $\mathrm{s}$ " shape as the contact area decreases. The shape of curve $c$ shows a typical electrochemical behavior of microelectrodes.

Two methods were applied to calculate the electrode area, i.e., image analysis method and electrochemical calculation method. The relevant equations can be written as

$$
\begin{aligned}
& i_{\mathrm{ss}}=4 n F D C r \\
& i_{\mathrm{p}}=2.69 \times 10^{5} n^{3 / 2} A C D^{1 / 2} v^{1 / 2}
\end{aligned}
$$

where $i_{\text {ss }}$ is the steady-state limit diffusion current, $n$ represents the transfer electron number, $F$ denotes Faraday constant, $D$ and $C$ are the diffusion coefficient and concentration of the reaction medium, respectively, $r$ is the radius of the microelectrode, $i_{\mathrm{p}}$ is peak current, and $A$ and $v$ are the effective electrode active area and the scanning rate, respectively. The 


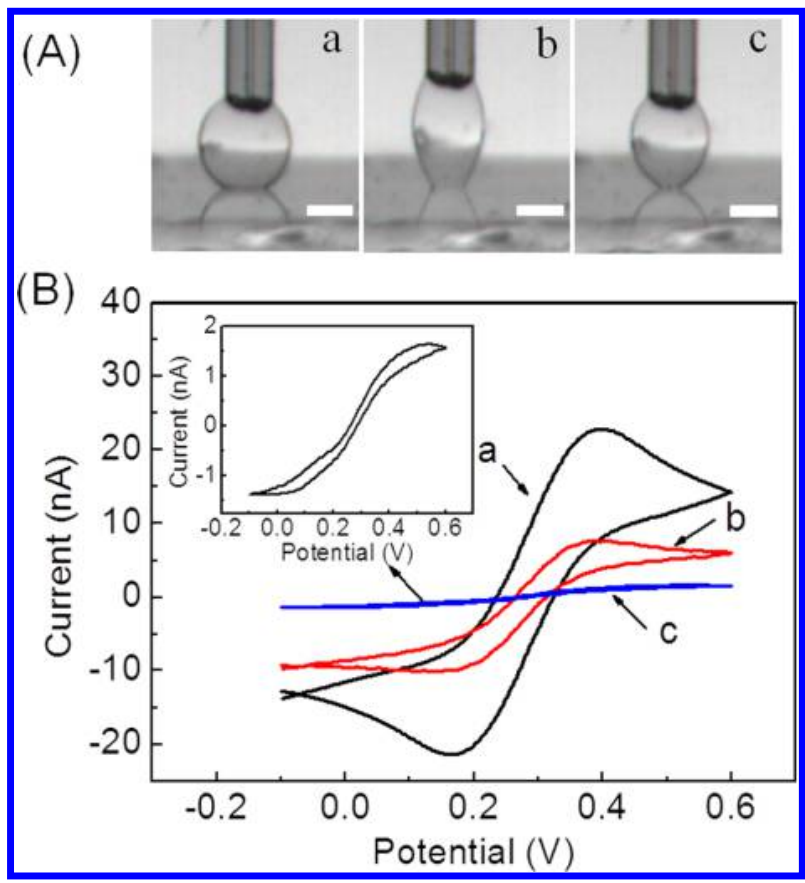

Figure 2. (A) Three different states of droplets on the TODS selfassembled monolayer-modified ITO electrode and (B) the corresponding CV curves in $1 \mathrm{mM} \mathrm{K} \mathrm{K}_{3} \mathrm{Fe}(\mathrm{CN})_{6}$ with a scanning rate of 20 $\mathrm{mV} / \mathrm{s}$. The scale bar is $372 \mu \mathrm{m}$.

reaction medium in this system is $\mathrm{K}_{3} \mathrm{Fe}(\mathrm{CN})_{6} \cdot\left[\mathrm{Fe}(\mathrm{CN})_{6}\right]^{3-}$ is a good reversible redox species; thus, the diffusion coefficient of
$\mathrm{K}_{3} \mathrm{Fe}(\mathrm{CN})_{6}$ in $2.5 \mathrm{M} \mathrm{KCl}(D)$ can be calculated by the relationship of peak current and reaction medium activity in the reversible system (eq 2). CV curves were measured at different scan rates $(30 \mathrm{mV} / \mathrm{s}, 50 \mathrm{mV} / \mathrm{s}, 70 \mathrm{mV} / \mathrm{s}, 100 \mathrm{mV} / \mathrm{s}, 120 \mathrm{mV} / \mathrm{s})$ with fixed ITO electrode size $(R=2.5 \mathrm{~mm})$, as shown in Figure S2a, Supporting Information. From the linear relation of $i_{\mathrm{p}}$ and $v_{1 / 2}$ (Figure S2b, Supporting Information), $D$ was determined to be $2.439 \times 10^{-6} \mathrm{~cm}^{2} / \mathrm{s}$ according to eq 2 .

Curves $\mathrm{a}$ and $\mathrm{b}$ can be attributed to the conventional size electrode because there are obvious redox peaks in the $\mathrm{CV}$ curves; thus, the relationship of electrode size and peak current is satisfied with eq 2. Curve $c$ is close to a sigmoid curve where the electrode size can be calculated using eq 1 . The electrode size of states $a, b$, and $c$ obtained using the image analysis method is $124.3,60.8$, and $29.5 \mu \mathrm{m}$, respectively, while the corresponding size calculated by the electrochemical calculation method is $141.9,82.1$, and $34.6 \mu \mathrm{m}$, respectively. The values obtained using the latter method are larger than those obtained from image analysis, which is probably due to the roughness of the ITO surfaces. The electrode size can be adjusted down to $\sim 30 \mu \mathrm{m}$ in radius.

Electrochemical Characterizations of DSLM on Bare ITO Electrodes. The electrochemical technique is well-known to characterize bilayers. ${ }^{9,15,31}$ The formation of DSLM on the hydrophilic surface of the ITO electrode was characterized by cyclic voltammetry. Figure $3 \mathrm{~A}(\mathrm{a}, \mathrm{b})$ are the normalized $\mathrm{CV}$ curves of the bare ITO electrode and lipid bilayer modified electrode, respectively. After the formation of lipid bilayer membranes on the surface of the ITO electrode, the current decreased to $\mathrm{pA}$ magnitude and the redox peaks disappeared

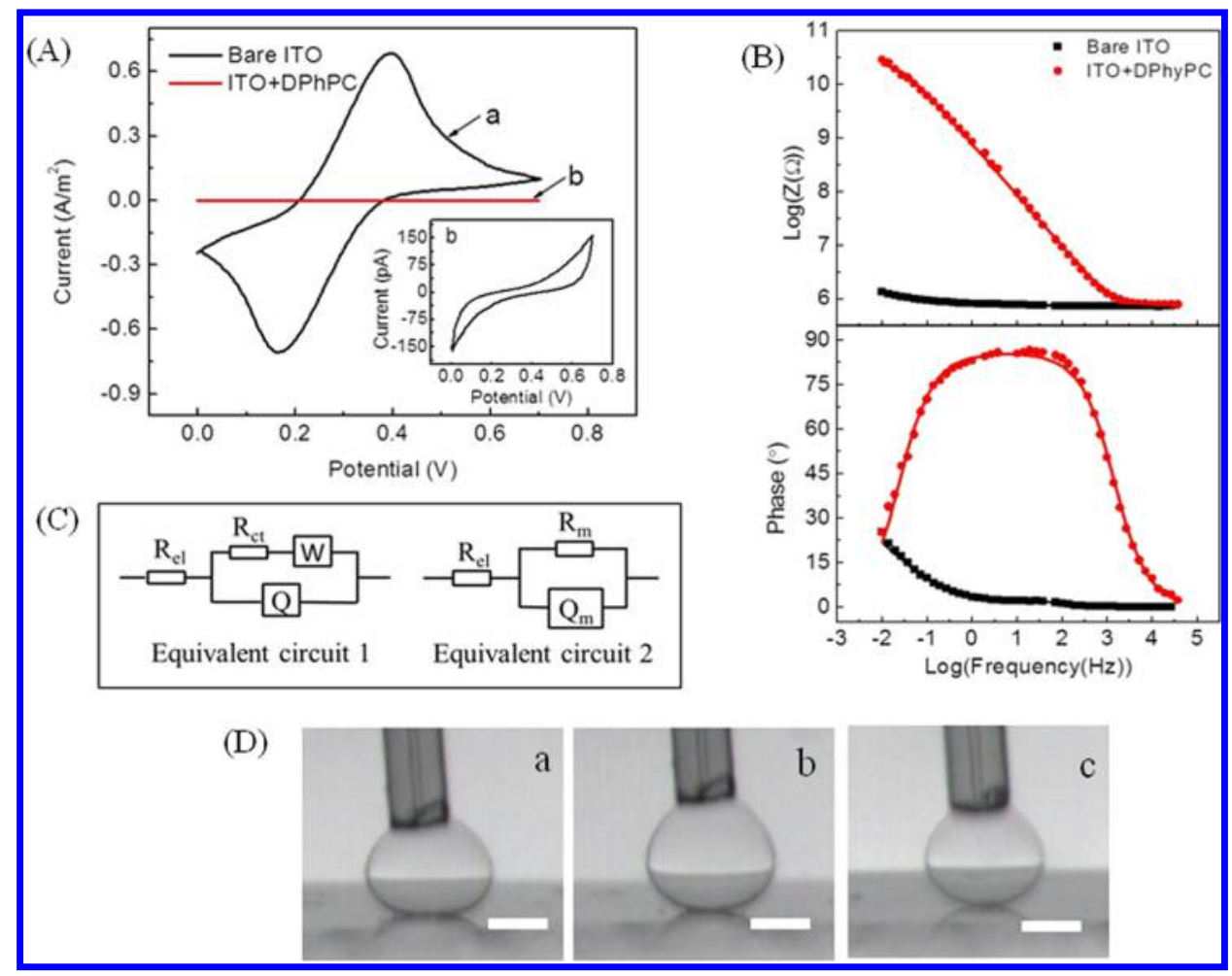

Figure 3. (A) CV curves of the bare ITO electrode and the lipid bilayer membrane-modified ITO electrode in $0.5 \mathrm{mM} \mathrm{K}_{3} \mathrm{Fe}(\mathrm{CN})_{6}$ solution containing $0.2 \mathrm{M}$ PBS buffer and $2 \mathrm{M} \mathrm{KCl}$. The scanning rate is $10 \mathrm{mV} / \mathrm{s}$. The inset is the CV curve of the lipid bilayer membrane-modified ITO electrode. (B) Impedance spectra of bare ITO (square symbols) and after the addition of DPhyPC (rhombus symbols) in hexane at $0.2 \mathrm{~V}$ bias in 0.2 M PBS buffer containing $0.5 \mathrm{mM} \mathrm{K}_{3} \mathrm{Fe}(\mathrm{CN})_{6}$ and $2 \mathrm{M} \mathrm{KCl}$. The solid lines are the fitting results using the corresponding equivalent circuits in $\mathrm{C}$. (D) Three different states of droplets on the ITO electrode in hexane with DPhyPC. The scale bar is $372 \mu \mathrm{m}$. 
completely, as shown in the inset in Figure 3A. The decrease of peak current and shape change of curve $b$ compared with curve a indicate the existence of lipid bilayer membranes which hinder the charge transfer of $\left[\mathrm{Fe}(\mathrm{CN})_{6}\right]^{3-}$.

To investigate the lipid bilayer membrane more quantitatively, the electrochemical impedance spectroscopy (EIS) technique was employed in a frequency regime of $10^{-2}$ to $10^{5} \mathrm{~Hz}$. Impedance spectra of bare ITO (square symbols) before and after the addition of lipid (dot symbols) in hexane are shown in Figure 3B. The capacitance of the DPhyPC bilayer $\left(Q_{\mathrm{m}}\right)$ is $0.4 \mu \mathrm{F} \mathrm{cm}{ }^{-2}(n=0.954)$, which is in the range of the characteristic capacitance of lipid bilayers (0.4-0.9 $\mu \mathrm{F}$ $\left.\mathrm{cm}^{-2}\right) .{ }^{13,32}$ The impedance increases dramatically after addition of the lipid (DPhyPC) in hexane, which clearly indicates the formation of an insulating layer at the contact domain between the droplet and the surface of the ITO electrode. Equivalent circuits 1 and 2 in Figure 3C were used to fit the bare ITO electrode and the lipid bilayer-modified electrode, respectively. $R_{\mathrm{el}}, R_{\mathrm{ct}}$ and $W$ represent the resistance of the electrolyte solution, the charge transfer resistance, and the Warburg impedance denoting the diffusion of $\left[\mathrm{Fe}(\mathrm{CN})_{6}\right]^{3-}$, respectively; $R_{\mathrm{m}}$ represents the resistance of DPhyPC lipid bilayer membranes. The ideal capacitances are replaced by the constant-phase element (CPE), taking into account the frequency dispersion of the capacitance due to factors such as inhomogeneity of the surface. The constant-phase element can be identified as $Z_{\mathrm{CPE}}(\omega)=1 / j(\omega \mathrm{Q})^{n}$, where $n(0<n<1)$ is the CPE exponent. CPE can be regarded as a pure capacitance when $n$ is close to 1 , and a pure resistance when $n$ is close to 0 . $Q$ in equivalent circuit 1 represents the capacitance of the electric double layer at the surface of the ITO electrode. $Q_{m}$ in equivalent circuit 2 represents the capacitance of lipid bilayer membranes. Impedance data can be fitted very well using these two equivalent circuits as shown in Figure 3B. The membrane resistance of the DPhyPC bilayer $\left(R_{\mathrm{m}}\right)$ is $26.3 \mathrm{G} \Omega$. The normalized resistivity calculated from the contact area is $15 \mathrm{M} \Omega$ $\mathrm{cm}^{2}$, which is 3 orders of magnitude higher than that of conventional supported lipid membranes. ${ }^{33,34}$ This value is in the same magnitude and even higher than that of the tethered lipid bilayer membranes on gold surfaces. ${ }^{35,36}$ Compared with the gigasealing lipid bilayer on a single polyelectrolyte multilayer-filled nanopore, ${ }^{19}$ the resistivity is 6 orders of magnitude higher than that in their research $\left(5 \Omega \mathrm{cm}^{2}\right)$, which implies that there are nearly no defects in our gigasealing lipid bilayer. The droplet-solid interface lipid bilayer membranes can remain intact and stable as long as the volatilization of hexane with lipid is prevented.

The area of the DSLM can be easily controlled, as shown in Figure 3D. The membrane radius of states a, b, and c obtained using the image analysis method is $170.5,105.2$, and $58.9 \mu \mathrm{m}$, respectively. The corresponding CV curves are shown in Figure S3, Supporting Information. The current is in pA magnitude and decreases gradually as the contact area decreases, which indicates that DSLM is suitable for ion channel analysis.

Lateral Mobility of the Lipid Bilayer. The lateral diffusion coefficient is a vital parameter to characterize the fluidity of the supported lipid bilayer. As an excellent approach to characterize the lateral fluidity, fluorescence recovery after photobleaching (FRAP) was applied to measure the diffusion coefficient $D$ of the Texas Red DHPE (0.5\%) in the lipid (egg PC) bilayer formed at the contact domain between the aqueous droplet and the bare ITO electrode (Figure 4). The images in Figure $4 \mathrm{a}, \mathrm{b}$ were taken at $10 \mathrm{~s}$ and $19 \mathrm{~min}$ after photobleaching,

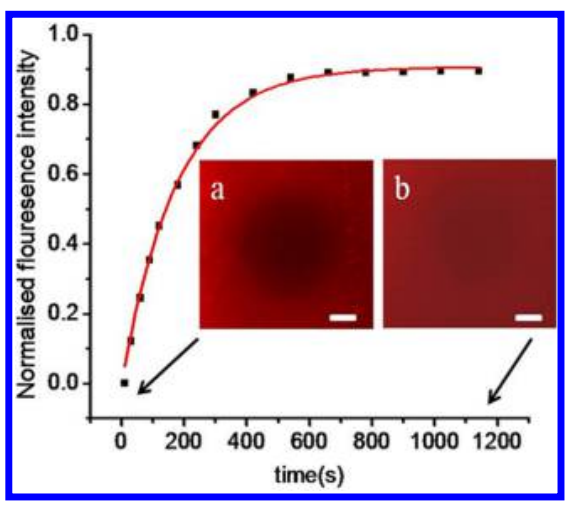

Figure 4. FRAP experiment on lipid bilayer formed at the contact domain between the droplet and the bare ITO support. Fluorescence images of the bilayer were taken at (a) $10 \mathrm{~s}$ and (b) 19 min after photobleaching. Scale bar is $20 \mu \mathrm{m}$.

respectively. The fluorescence intensity of the dark spot taken at $10 \mathrm{~s}$ after photobleaching recovered homogeneously after 19 min. The coefficient $D$ value was calculated to be $2.58 \mu \mathrm{m}^{2} \mathrm{~s}^{-1}$ with a mobile fraction of 0.9. The FRAP experiments confirm excellent mobility of the lipids in the bilayers, which is important for further membrane protein studies.

Interaction of Melittin with Lipid Bilayers. To test the validity of the obtained high impedance DSLM for observing peptide channel activities, melittin was chosen for the study. As the principal toxic component of bee venom, melittin is a cationic, hemolytic peptide ${ }^{37,38}$ composed of 26 amino acids residues in which the amino-terminal region is predominantly hydrophobic whereas the carboxyl-terminal region is hydrophilic due to the presence of a stretch of positively charged amino acids. ${ }^{39}$ This amphiphilic property has resulted in melittin being used as a suitable model peptide for monitoring lipid-protein interactions in membranes. Melittin induces transmembrane pores that conform to the toroidal model through monomer bending of the external monolayer sheet via strong ionic interactions with the lipid headgroups. ${ }^{40}$

Time-dependent experiments for the melittin and membrane interactions have proven to be very sensitive in the process of pore formation. ${ }^{41-43} \mathrm{We}$ measured the membrane resistance and capacitance of the lipid bilayer to monitor the pore formation process of melittin. Figure 5 shows the variation of membrane resistance and capacitance against time after the addition of $1 \mu \mathrm{M}$ melittin to the lipid bilayer. The gradual formation of pores leads to the gradual decrease of the membrane resistance due to the binding of melittin monomers to the membrane and oligomerization. Simultaneously, the

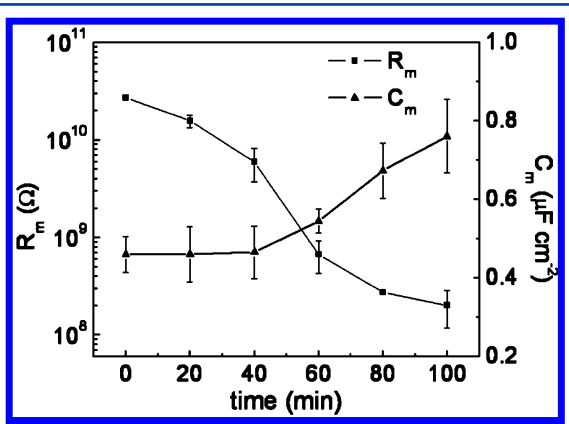

Figure 5. Time course of membrane resistance and capacitance of the DPhyPC bilayer in the presence of $1 \mu \mathrm{M}$ melittin. 
membrane capacitance remained unchanged $\left(\sim 0.46 \mu \mathrm{F} \mathrm{cm}^{-2}\right)$ during the first $40 \mathrm{~min}$ and increased to $0.76 \mu \mathrm{F} \mathrm{cm} \mathrm{cm}^{-2}$ in 100 min, which can be attributed to an increase in the mean dielectric constant due to a larger amount of water $\left(\varepsilon_{\mathrm{r}} \approx 80\right)$ within the lipid bilayer $\left(\varepsilon_{\mathrm{r}} \approx 2-3\right){ }^{43}$ The fitting values of membrane capacitance were all in the range of the characteristic capacitance of lipid bilayers (0.4-0.9 $\mu \mathrm{F} \mathrm{cm} \mathrm{cm}^{-2}$ ), which demonstrated that the lipid bilayer was still intact $100 \mathrm{~min}$ after the addition of $1 \mu \mathrm{M}$ melittin.

Besides the time-independent experiments, the interaction between the lipid bilayer and melittin at different concentrations was studied. Figure 6 shows the impedance spectra of

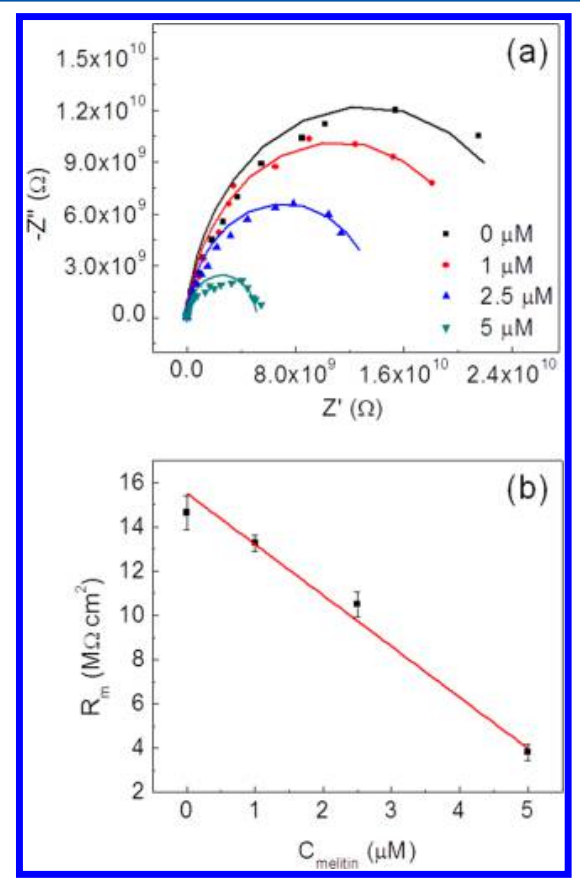

Figure 6. (a) Electrochemical impedance spectra (dotted plots) of the DPhyPC bilayer before and after interacting with melittin at different concentrations $(1,2.5$, and $5 \mu \mathrm{M})$. The solid lines are fitting curves using the appropriate equivalent circuit. (b) The membrane resistance as a function of melittin concentration.

the DPhyPC bilayer before and after interacting with melittin at different concentrations and the relationship between membrane resistance and melittin concentration. Melittin was dissolved in 0.2 M PBS containing $0.5 \mathrm{mM} \mathrm{K}_{3} \mathrm{Fe}(\mathrm{CN})_{6}$, and $2 \mathrm{M} \mathrm{KCl}$. Impedance spectra were fitted using the equivalent circuit 2 shown in Figure 3C, which consists of solution resistance $R_{\mathrm{el}}$ in series with the membrane resistance and capacitance, $R_{\mathrm{m}}$ and $Q$ respectively. The parameters obtained from the fitting were summarized in Table 1 . We have known that the membrane resistance decreased with prolonged time after the addition of melittin at a given concentration. Thus, all the EIS measurements were performed 20 min later, after the addition of melittin, to provide enough time for the gradual

\section{Table 1. EIS Fitting Parameters for Data in Figure 6}

$\begin{array}{cccc}C \text { (melittin) } / \mu \mathrm{M} & R_{\mathrm{m}} / \mathrm{M} \Omega \mathrm{cm}^{2} & C_{\mathrm{m}} / \mu \mathrm{F} \mathrm{cm} & N \\ 0 & 14.6 & 0.39 & 0.954 \\ 1 & 13.2 & 0.46 & 0.942 \\ 2.5 & 10.7 & 0.41 & 0.950 \\ 5 & 3.7 & 0.35 & 0.971\end{array}$

formation of channels on the lipid bilayer membranes. The $R_{\mathrm{m}}$ values were determined to be $13.2,10.7$, and $3.7 \mathrm{M} \Omega \mathrm{cm}^{2}$ at melittin concentrations of $1,2.5$, and $5 \mu \mathrm{M}$, respectively. The membrane resistance decreases linearly with the increase of melittin concentration $\left(R^{2}=0.988\right)$. Throughout the experiment, the membrane capacitance remained relatively unchanged, which illustrated that the addition of melittin did not destroy the lipid bilayer membrane.

\section{CONCLUSIONS}

A novel droplet-solid support lipid bilayer membrane (DLSM) system with high impedance was fabricated through controlling the contact area between the droplet and electrode. Our DLSM system combined traditional SLBs and novel DIBs, resulting in several characteristics. First, the lipid bilayer membrane resistance prepared in this method can reach up to $G \Omega$ magnitude, which allows for melittin ion channel behavior study. Second, our setup is transparent, which is convenient for the optical research; thus, the electrochemical method and photological method can be applied simultaneously with our system. Third, the DLSM system owns good fluidity. Fourth, compared with the patch clamp technique, the sensitivity of protein detection with the electrochemical method is lower; however, the electrochemical method can be accessed more easily and is more popular than the patch clamp technique. The lack of a reservoir beneath the membrane may limit its reconstitution of transmembrane proteins. Overall, the droplet-solid interface lipid membranes are flexible, stable, and low cost. This artificial cell model system has great potential for ion channel studies and high-throughput drug screening.

\section{ASSOCIATED CONTENT}

\section{S Supporting Information}

Calibration of $\mathrm{Ag} / \mathrm{AgCl}$ electrode, $\mathrm{CV}$ curves to calculate the diffusion coefficient of $\mathrm{K}_{3} \mathrm{Fe}(\mathrm{CN})_{6}$ in $2.5 \mathrm{M} \mathrm{KCl}(D)$, and $\mathrm{CV}$ curves corresponding to the droplet situation $a, b$, and $c$ in Figure 3D. This material is available free of charge via the Internet at http://pubs.acs.org.

\section{AUTHOR INFORMATION}

\section{Corresponding Author}

*E-mail: hanxiaojun@hit.edu.cn.

\section{Notes}

The authors declare no competing financial interest.

\section{ACKNOWLEDGMENTS}

This work was supported by the National Natural Science Foundation of China (grant nos. 21273059, 21003032, 21103214), State Key Laboratory of Urban Water Resource and Environment (Harbin Institute of Technology) (grant no. 2014DX09), Harbin Science and Technology Research Council (grant no. 2014RFXXJ063), and the Research Program of Qingdao (12-1-4-9-(4)-jch).

\section{REFERENCES}

(1) Drews, J. Science 2000, 287, 1960.

(2) Liu, A.; Zhao, Q.; Krishantha, D. M.; Guan, X. J. Phys. Chem. Lett. 2011, 2, 1372.

(3) Bayley, H.; Cremer, P. S. Nature 2001, 413, 226.

(4) Ervin, E. N.; Kawano, R.; White, R. J.; White, H. S. Anal. Chem. 2008, 80, 2069. 
(5) Lathrop, D. K.; Ervin, E. N.; Barrall, G. A.; Keehan, M. G.; Kawano, R.; Krupka, M. A.; White, H. S.; Hibbs, A. H. J. Am. Chem. Soc. 2010, 132, 1878.

(6) Pawlak, M.; Stankowski, S.; Schwarz, G. Biochim. Biophys. Acta 1991, 1062, 94.

(7) Gomezlagunas, F.; Pena, A.; Lievano, A.; Darszon, A. Biophys. J. 1989, 56, 115.

(8) Bradley, R. J.; Urry, D. W.; Okamoto, K.; Rapaka, R. Science 1978, 200, 435.

(9) Hennesthal, C.; Steinem, C. I. Am. Chem. Soc. 2000, 122, 8085.

(10) Drexler, J.; Steinem, C. I. Phvs. Chem. B 2003, 107, 11245.

(11) Horn, C.; Steinem, C. Biophvs. I. 2005, 89, 1046.

(12) Venkatesan, B. M.; Polans, J.; Comer, J.; Sridhar, S.; Wendell,

D.; Aksimentiev, A.; Bashir, R. Biomed. Microdevices 2011, 13, 671.

(13) Romer, W.; Steinem, C. Biophvs. I. 2004, 86, 955.

(14) Schmitt, E. K.; Vrouenraets, M.; Steinem, C. Biophys. J. 2006, 91, 2163.

(15) Han, X.; Studer, A.; Sehr, H.; Geissbühler, I.; Di Berardino, M.; Winkler, F. K.; Tiefenauer, L. X. Adv. Mater. 2007, 19, 4466.

(16) Zhu, Z. W.; Wang, Y.; Zhang, X.; Sun, C. F.; Li, M. G.; Yan, J. W.; Mao, B. W. Langmuir 2012, 28, 14739.

(17) Tantawi, K. H.; Berdiev, B.; Cerro, R.; Williams, J. D. Superlattice Microstruct. 2013, 58, 72.

(18) Romer, W.; Lam, Y. H.; Fischer, D.; Watts, A.; Fischer, W. B.; Goring, P.; Wehrspohn, R. B.; Gosele, U.; Steinem, C. J. Am. Chem. Soc. 2004, 126, 16267.

(19) Sugihara, K.; Voros, J.; Zambelli, T. ACS Nano 2010, 4, 5047.

(20) Zhou, X. J.; Moran-Mirabal, J. M.; Craighead, H. G.; McEuen, P. L. Nat. Nanotechnol. 2007, 2, 185.

(21) Ang, P. K.; Loh, K. P.; Wohland, T.; Nesladek, M.; Van Hove, E. Adv. Funct. Mater. 2009, 19, 109.

(22) Yang, T. H.; Yee, C. K.; Amweg, M. L.; Singh, S.; Kendall, E. L.; Dattelbaum, A. M.; Shreve, A. P.; Brinker, C. J.; Parikh, A. N. Nano Lett. 2007, 7, 2446.

(23) Reimhult, E.; Zach, M.; Hook, F.; Kasemo, B. Langmuir 2006, 22, 3313.

(24) Andersson, M.; Keizer, H. M.; Zhu, C. Y.; Fine, D.; Dodabalapur, A.; Duran, R. S. Langmuir 2007, 23, 2924.

(25) Cornell, B. A.; BraachMaksvytis, V. L. B.; King, L. G.; Osman, P. D. J.; Raguse, B.; Wieczorek, L.; Pace, R. I. Nature 1997, 387, 580.

(26) Bayley, H.; Cronin, B.; Heron, A.; Holden, M. A.; Hwang, W. L.; Syeda, R.; Thompson, J.; Wallace, M. Mol. Biosyst. 2008, 4, 1191.

(27) Leptihn, S.; Castell, O. K.; Cronin, B.; Lee, E. H.; Gross, L. C. M.; Marshall, D. P.; Thompson, J. R.; Holden, M.; Wallace, M. I. Nat. Protoc. 2013, 8, 1048.

(28) Heron, A. J.; Thompson, J. R.; Mason, A. E.; Wallace, M. I. J. Am. Chem. Soc. 2007, 129, 16042.

(29) Soumpasis, D. M. Biophvs. I. 1983, 41, 95.

(30) Zhang, L. Q.; Longo, M. L.; Stroeve, P. Langmuir 2000, 16, 5093.

(31) Cheng, W. L.; Han, X. J.; Wang, E. K.; Dong, S. J. Electroanalysis 2004, 16, 127.

(32) Montal, M.; Mueller, P. Proc. Natl. Acad. Sci. U. S. A. 1972, 69, 3561 .

(33) Gritsch, S.; Nollert, P.; Jahnig, F.; Sackmann, E. Langmuir 1998, 14, 3118.

(34) Wiegand, G.; Arribas-Layton, N.; Hillebrandt, H.; Sackmann, E.; Wagner, P. I. Phvs. Chem. B 2002, 106, 4245.

(35) Schiller, S. M.; Naumann, R.; Lovejoy, K.; Kunz, H.; Knoll, W. Angew. Chem. Int. Ed. 2003, 42, 208.

(36) He, L. H.; Robertson, J. W. F.; Li, J.; Karcher, I.; Schiller, S. M.; Knoll, W.; Naumann, R. Lanomuir 2005, 21, 11666.

(37) Habermann, E. Science 1972, 177, 314.

(38) Dempsey, C. E. Biochim. Biophys. Acta 1990, 1031, 143.

(39) Raghuraman, H.; Chattopadhyay, A. Biosci. Rep. 2007, 27, 189.

(40) Yang, L.; Harroun, T. A.; Weiss, T. M.; Ding, L.; Huang, H. W. Biophvs. I. 2001, 81, 1475.

(41) Studer, A.; Han, X. J.; Winkler, F. K.; Tiefenauer, L. X. Colloids Surf. B 2009, 73, 325.
(42) Matsuzaki, K.; Yoneyama, S.; Miyajima, K. Biophvs. I. 1997, 73, 831.

(43) Steinem, C.; Galla, H. J.; Janshoff, A. Phys. Chem. Chem. Phys. 2000, 2, 4580. 